\title{
On the M-Projective Curvature Tensor of $N(\kappa)$-Contact Metric Manifolds
}

\author{
R. N. Singh and Shravan K. Pandey \\ Department of Mathematical Sciences, APS University, Rewa, Madhya Pradesh 486003, India \\ Correspondence should be addressed to Shravan K. Pandey; shravan.math@gmail.com
}

Received 29 December 2012; Accepted 20 January 2013

Academic Editors: J. Keesling, A. Morozov, and E. Previato

Copyright (C) 2013 R. N. Singh and S. K. Pandey. This is an open access article distributed under the Creative Commons Attribution License, which permits unrestricted use, distribution, and reproduction in any medium, provided the original work is properly cited.

The object of the present paper is to study some curvature conditions on $N(\kappa)$-contact metric manifolds.

\section{Introduction}

The notion of the odd dimensional manifolds with contact and almost contact structures was initiated by Boothby and Wong in 1958 rather from topological point of view. Sasaki and Hatakeyama reinvestigated them using tensor calculus in 1961. Tanno [1] classified the connected almost contact metric manifolds whose automorphism groups possess the maximum dimension. For such a manifold, the sectional curvature of plain sections containing $\xi$ is a constant, say $c$. He showed that they can be divided into three classes: (i) homogeneous normal contact Riemannian manifolds with $c>0$, (ii) global Riemannian products of line or a circle with a Kähler manifold of constant holomorphic sectional curvature if $c=0$, and (iii) a warped product space $\mathbb{R} \times{ }_{f} \mathbb{C}^{n}$ if $c<0$. It is known that the manifolds of class (i) are characterized by admitting a Sasakian structure. Kenmotsu [2] characterized the differential geometric properties of the manifolds of class (iii); so the structure obtained is now known as Kenmotsu structure. In general, these structures are not Sasakian [2].

On the other hand in Pokhariyal and Mishra [3] defined a tensor field $W^{*}$ on a Riemannian manifold as

$$
\begin{aligned}
& { }^{\prime} W^{*}(X, Y, Z, U) \\
& ={ }^{\prime} R(X, Y, Z, U)-\frac{1}{2(n-1)} \\
& \quad \times[S(Y, Z) g(X, U)
\end{aligned}
$$

$$
\begin{aligned}
& -S(X, Z) g(Y, U)+g(Y, Z) S(X, U) \\
& -g(X, Z) S(Y, U)]
\end{aligned}
$$

where ${ }^{\prime} W^{*}(X, Y, Z, U)=g\left(W^{*}(X, Y) Z, U\right)$ and ${ }^{\prime} R(X, Y$, $Z, U)=g(R(X, Y) Z, U)$. Such a tensor field $W^{*}$ is known as $\mathrm{m}$-projective curvature tensor. Later, Ojha [4] defined and studied the properties of $\mathrm{m}$-projective curvature tensor in Sasakian and Kähler manifolds. He also showed that it bridges the gap between the conformal curvature tensor, conharmonic curvature tensor, and concircular curvature tensor on one side and $\mathrm{H}$-projective curvature tensor on the other. Recently m-projective curvature tensor has been studied by Chaubey and Ojha [5], Singh et al. [6], Singh [7], and many others. Motivated by the above studies, in the present paper, we study flatness and symmetry property of $N(\kappa)$-contact metric manifolds regarding m-projective curvature tensor. The present paper is organized as follows.

In this paper, we study the $\mathrm{m}$-projective curvature tensor of $N(\kappa)$-contact metric manifolds. In Section 2, some preliminary results are recalled. In Section 3, we study $\mathrm{m}$-projectively semisymmetric $N(\kappa)$-contact metric manifolds. Section 4 deals with m-projectively flat $N(\kappa)$-contact metric manifolds. $\xi$-m-projectively flat $N(\kappa)$-contact metric manifolds are studied in Section 5 and obtained necessary and sufficient condition for an $N(\kappa)$-contact metric manifold to be $\xi$-mprojectively flat. In Section 6, m-projectively recurrent $N(\kappa)$ contact metric manifolds are studied. Section 7 is devoted to the study of $N(\kappa)$-contact metric manifolds satisfying 
$W^{*} \cdot S=0$. The last section deals with an $N(\kappa)$-contact metric manifolds satisfying $W^{*} \cdot R=0$.

\section{Contact Metric Manifolds}

An odd dimensional differentiable manifold $M^{2 n+1}$ is said to admit an almost contact structure if there exist a tensor field $\phi$ of type $(1,1)$, a vector field $\xi$, and a 1-form $\eta$ satisfying

$$
\begin{gathered}
\phi^{2}=-I+\eta \otimes \xi, \\
\eta(\xi)=1, \\
\phi \xi=0, \\
\eta \circ \phi=0 .
\end{gathered}
$$

An almost contact metric structure is said to be normal if the induced almost complex structure $J$ on the product manifold $M^{2 n+1} \times \mathbb{R}$ defined by

$$
J\left(X, f \frac{d}{d t}\right)=\left(\phi X-f \xi, \eta(X) \frac{d}{d t}\right)
$$

is integrable, where $X$ is tangent to $M^{2 n+1}, t$ is coordinate of $\mathbb{R}$, and $f$ is smooth function on $M^{2 n+1} \times \mathbb{R}$. Let $g$ be a compatible Riemannian metric with almost contact structure $(\phi, \xi, \eta)$, that is,

$$
g(\phi X, \phi Y)=g(X, Y)-\eta(X) \eta(Y) .
$$

Then, $M^{2 n+1}$ becomes an almost contact metric manifold equipped with an almost contact metric structure $(\phi, \xi, \eta, g)$. From (2) and (7), it can be easily seen that

$$
\begin{gathered}
g(X, \phi Y)=-g(\phi X, Y), \\
g(X, \xi)=\eta(X),
\end{gathered}
$$

for all vector fields $X$ and $Y$. An almost contact metric structure becomes a contact metric structure if

$$
g(X, \phi Y)=d \eta(X, Y),
$$

for all vector fields $X$ and $Y$. The 1 -form $\eta$ is then a contact form, and $\xi$ is its characteristic vector field. We define a $(1,1)$ tensor field $h$ by $h=(1 / 2) \mathfrak{E}_{\xi} \phi$, where $£$ denotes the Liedifferentiation. Then, $h$ is symmetric and satisfies $h \phi=-\phi h$. We have $\operatorname{Tr} \cdot h=\operatorname{Tr} \cdot \phi h=0$ and $h \xi=0$. Also,

$$
\nabla_{X} \xi=-\phi X-\phi h X
$$

holds in a contact metric manifold.

A normal contact metric manifold is a Sasakian manifold. An almost contact metric manifold is Sasakian if and only if

$$
\left(\nabla_{X} \phi\right)(Y)=g(X, Y) \xi-\eta(Y) X,
$$

for all vector fields $X$ and $Y$, where $\nabla$ is the Levi-Civita connection of the Riemannian metric $g$. A contact metric manifold $M^{2 n+1}$ for which $\xi$ is a killing vector is said to be a K-contact manifold. A Sasakian manifold is K-contact, but the converse needs not be true. However, a 3-dimensional Kcontact manifold is Sasakian [8]. It is well known that the tangent sphere bundle of a flat Riemannian manifold admits a contact metric structure satisfying $R(X, Y) \xi=0$ [9]. On the other hand, on a Sasakian manifold the following holds:

$$
R(X, Y) \xi=\eta(Y) X-\eta(X) Y .
$$

As a generalization of both $R(X, Y) \xi=0$ and the Sasakian case; Blair et al. [11] considered the $(\kappa, \mu)$-nullity condition on a contact metric manifold and gave several reasons for studying it.

The $(\kappa, \mu)$-nullity distribution $N(\kappa, \mu)([10,11])$ of contact metric manifold is defined by

$$
\begin{aligned}
N(\kappa, \mu): p & \longrightarrow N_{p}(\kappa, \mu) \\
=\left\{Z \in T_{p} M: R(X, Y) Z\right. & =(\kappa I+\mu h)[g(Y, Z) X-g(X, Z) Y]\},
\end{aligned}
$$

for all $X, Y \in T M$, where $(\kappa, \mu) \in \mathbb{R}^{2}$. A contact metric manifold $M^{2 n+1}$ with $\xi \in N(\kappa, \mu)$ is called a $(\kappa, \mu)$-manifold. In particular, on a $(\kappa, \mu)$-manifold, we have

$$
\begin{aligned}
R(X, Y) \xi= & \kappa[\eta(Y) X-\eta(X) Y] \\
& +\mu[\eta(Y) h X-\eta(X) h Y] .
\end{aligned}
$$

On a $(\kappa, \mu)$-manifold, $\kappa \leq 1$. If $\kappa=1$, the structure is Sasakian ( $h=0$ and $\mu$ is indeterminate), and if $\kappa<1$, the $(\kappa, \mu)$-nullity condition determines the curvature of $M^{2 n+1}$ completely [11]. In fact, for a $(\kappa, \mu)$-manifold, the conditions of being a Sasakian manifold, a K-contact manifold, $\kappa=1$ and $h=0$ are all equivalent.

In a $(\kappa, \mu)$-manifold, the following relations hold $([11,12])$ :

$$
\begin{gathered}
h^{2}=(\kappa-1)^{2} \phi^{2}, \quad \kappa \leq 1, \\
\left(\nabla_{X} \phi\right)(Y)=g(X+h X, Y) \xi-\eta(Y)(X+h X), \\
R(\xi, X) Y=\kappa[g(X, Y) \xi-\eta(Y) X] \\
+\mu[g(h X, Y) \xi-\eta(Y) h X], \\
S(X, \xi)=2 n \kappa \eta(X), \\
S(X, Y)=[2(n-1)-n \mu] g(X, Y) \\
+[2(n-1)+\mu] g(h X, Y) \\
+[2(1-n)+n(2 \kappa+\mu)] \eta(X) \eta(Y), \\
r=2 n(2 n-2+\kappa-n \mu), \\
S(\phi X, \phi Y)=S(X, Y)-2 n \kappa \eta(X) \eta(Y) \\
-2(2 n-2+\mu) g(h X, Y),
\end{gathered}
$$


where $S$ is the Ricci tensor of type $(0,2), Q$ is the Ricci operator, that is, $g(Q X, Y)=S(X, Y)$, and $r$ is the scalar curvature of the manifold. From (11), it follows that

$$
\left(\nabla_{X} \eta\right)(Y)=g(X+h X, \phi Y)
$$

Also in a $(\kappa, \mu)$-manifold,

$$
\begin{aligned}
\eta(R(X, Y) Z)= & \kappa[g(Y, Z) \eta(X)-g(X, Z) \eta(Y)] \\
& +\mu[g(h Y, Z) \eta(X)-g(h X, Z) \eta(Y)]
\end{aligned}
$$

holds.

The $\kappa$-nullity distribution $N(\kappa)$ of a Riemannian manifold $M^{2 n+1}[13]$ is defined by

$$
\begin{aligned}
N(\kappa): p \longrightarrow N_{p}(\kappa)=\{Z & \in T_{p} M: R(X, Y) Z \\
& =\kappa(g(Y, Z) X-g(X, Z) Y)\},
\end{aligned}
$$

for all $X, Y \in T M$ and $\kappa$ being a constant. If the characteristic vector field $\xi \in N(\kappa)$, then we call a contact metric manifold an $N(\kappa)$-contact metric manifold [14]. If $\kappa=1$, then $N(\kappa)$ contact metric manifold is Sasakian, and if $\kappa=0$, then $N(\kappa)$ contact metric manifold is locally isometric to the product $E^{n+1} \times S^{n}(4)$ for $n>1$ and flat for $n=1$. If $\kappa<1$, the scalar curvature is $r=2 n(2 n-2+\kappa)$. If $\mu=0$, then a $(\kappa, \mu)$-contact metric manifold reduces to a $N(\kappa)$-contact metric manifolds. In [9], $N(\kappa)$-contact metric manifold were studied in some detail.

In $N(\kappa)$-contact metric manifolds the following relations hold $([15,16])$ :

$$
\begin{gathered}
h^{2}=(\kappa-1) \phi^{2}, \quad \kappa \leq 1, \\
\left(\nabla_{X} \phi\right)(Y)=g(X+h X, Y) \xi-\eta(Y)(X+h X), \\
R(\xi, X) Y=\kappa[g(X, Y) \xi-\eta(Y) X], \\
S(X, \xi)=2 n \kappa \eta(X), \\
S(X, Y)=2(n-1)[g(X, Y)+g(h X, Y)] \\
+[2(1-n)+2 n \kappa] \eta(X) \eta(Y), \quad n \geq 1, \\
r=2 n(2 n-2+\kappa), \\
S(\phi X, \phi Y)=S(X, Y)-2 n \kappa \eta(X) \eta(Y) \\
-4(n-1) g(h X, Y), \\
\left(\nabla_{X} \eta\right)(Y)=g(X+h X, \phi Y), \\
R(X, Y) \xi=\kappa[\eta(Y) X-\eta(X) Y], \\
\eta(R(X, Y) Z)=\kappa[g(Y, Z) \eta(X)-g(X, Z) \eta(Y)] .
\end{gathered}
$$

For a $(2 n+1)$-dimensional $(n>1)$ almost contact metric manifold, m-projective curvature tensor $W^{*}$ is given by [3]

$$
\begin{aligned}
W^{*}(X, Y) Z= & R(X, Y) Z-\frac{1}{2(n-1)} \\
\times & {[S(Y, Z) X-S(X, Z) Y,} \\
& +g(Y, Z) Q X-g(X, Z) Q Y],
\end{aligned}
$$

for arbitrary vector fields $X, Y$, and $Z$, where $S$ is the Ricci tensor of type $(0,2)$ and $Q$ is the Ricci operator, that is, $g(Q X, Y)=S(X, Y)$.

The m-projective curvature tensor $W^{*}$ for an $N(\kappa)$ contact metric manifold is given by

$$
\begin{aligned}
& W^{*}(X, Y) \xi \\
& =-\frac{\kappa}{(n-1)}[\eta(Y) X-\eta(X) Y] \\
& -\frac{1}{2(n-1)}[\eta(Y) Q X-\eta(X) Q Y], \\
& \eta\left(W^{*}(X, Y) \xi\right)=0,
\end{aligned}
$$

$W^{*}(\xi, Y) Z$

$$
\begin{aligned}
= & -W^{*}(Y, \xi) Z \\
= & -\frac{\kappa}{(n-1)}[g(Y, Z) \xi-\eta(Z) Y] \\
& -\frac{1}{2(n-1)}[S(Y, Z) \xi-\eta(Z) Q Y],
\end{aligned}
$$

$\eta\left(W^{*}(\xi, Y) Z\right)$

$=-\eta\left(W^{*}(Y, \xi) Z\right)$

$$
=-\frac{\kappa}{(n-1)}[g(Y, Z)-\eta(Y) \eta(Z)]
$$

$$
-\frac{1}{2(n-1)}[S(Y, Z)-2 n \kappa \eta(Y) \eta(Z)],
$$

$$
\begin{aligned}
\eta( & \left.W^{*}(X, Y) Z\right) \\
= & -\frac{\kappa}{(n-1)}[g(Y, Z) \eta(X)-g(X, Z) \eta(Y)] \\
& -\frac{1}{2(n-1)}[S(Y, Z) \eta(X)-S(X, Z) \eta(Y)] .
\end{aligned}
$$

\section{M-Projectively Semisymmetric $N(\kappa)$-Contact Metric Manifolds}

Definition 1. A $(2 n+1)$-dimensional $(n>1) N(\kappa)$-contact metric manifold is said to be m-projectively semisymmetric [17] if it satisfies $R \cdot W^{*}=0$, where $R$ is the Riemannian curvature tensor and $W^{*}$ is the m-projective curvature tensor of the manifold.

Theorem 2. An m-projectively semisymmetric $N(\kappa)$-contact metric manifold is an Einstein manifold. 
Proof. Suppose that an $N(\kappa)$-contact metric manifold is mprojectively semisymmetric. Then, we have

$$
\left(R(\xi, X) \cdot W^{*}\right)(Y, Z) U=0 .
$$

The above equation can be written as follows:

$$
\begin{aligned}
& R(\xi, X) W^{*}(Y, Z) U-W^{*}(R(\xi, X) Y, Z) U \\
& \quad-W^{*}(Y, R(\xi, X) Z) U-W^{*}(Y, Z) R(\xi, X) U=0 .
\end{aligned}
$$

In view of (22), the above equation reduces to

$$
\begin{aligned}
\kappa & {\left[g\left(X, W^{*}(Y, Z) U\right) \xi-\eta\left(W^{*}(Y, Z) U\right) X\right.} \\
& -g(X, Y) W^{*}(\xi, Z) U+\eta(Y) W^{*}(X, Z) U \\
& -g(X, Z) W^{*}(Y, \xi) U+\eta(Z) W^{*}(Y, X) U \\
& \left.-g(X, U) W^{*}(Y, Z) \xi+\eta(U) W^{*}(Y, Z) X\right]=0 .
\end{aligned}
$$

Now, taking the inner product of the above equation with $\xi$ and using (3) and (9), we get

$$
\begin{aligned}
\kappa & {\left[{ }^{\prime} W^{*}(Y, Z, U, X)-\eta\left(W^{*}(Y, Z) U\right) \eta(X)\right.} \\
& -g(X, Y) \eta\left(W^{*}(\xi, Z) U\right)+\eta(Y) \eta\left(W^{*}(X, Z) U\right) \\
& -g(X, Z) \eta\left(W^{*}(Y, \xi) U\right)+\eta(Z) \eta\left(W^{*}(Y, X) U\right) \\
& \left.-g(X, U) \eta\left(W^{*}(Y, Z) \xi\right)+\eta(U) \eta\left(W^{*}(Y, Z) X\right)\right]=0,
\end{aligned}
$$

which on using (30), (32), (34), and (35), gives

$$
\begin{aligned}
\kappa\left[{ }^{\prime} R(Y, Z, U, X)-\frac{1}{2(n-1)}\right. \\
\quad \times\{S(Y, X) g(Z, U)-S(X, Z) g(Y, U) \\
\quad+S(X, Z) \eta(Y) \eta(U)-S(X, Y) \eta(Z) \eta(U)\} \\
+\frac{\kappa}{(n-1)} \\
\times\{g(Z, U) g(X, Y) \\
\quad-g(Y, U) g(X, Z)+n g(X, Z) \eta(Y) \eta(U) \\
\quad-n g(X, Y) \eta(Z) \eta(U)\}]=0 .
\end{aligned}
$$

Putting $Z=U=e_{i}$ in the above equation and taking summation over $i, 1 \leq i \leq 2 n+1$, we get

$$
S(X, Y)=2 n \kappa g(X, Y),
$$

which shows that $M^{2 n+1}$ is an Einstein manifold. This completes the proof.

\section{M-Projectively Flat $N(\kappa)$-Contact Metric Manifolds}

Theorem 3. An m-projectively flat $N(\kappa)$-contact metric manifold $M^{2 n+1}$ is an Einstein manifold.
Proof. Let $W^{*}(X, Y, Z, U)=0$. Then, from (30), we have

${ }^{\prime} R(X, Y, Z, U)$

$$
\begin{aligned}
=\frac{1}{2(n-1)}[ & S(Y, Z) g(X, U) \\
& -S(X, Z) g(Y, U)+g(Y, Z) S(X, U) \\
& -g(X, Z) g(Y, U)] .
\end{aligned}
$$

Let $\left\{e_{i}\right\}$ be an orthonormal basis of the tangent space at any point. Putting $Y=Z=e_{i}$ in the above equation and summing over $i, 1 \leq i \leq 2 n+1$, we get

$$
S(X, Y)=-r g(X, Y) \text {, }
$$

which shows that $M^{2 n+1}$ is an Einstein manifold. This completes the proof.

\section{5. $\xi$-M-Projectively Flat $N(\kappa)$-Contact Metric Manifolds}

Definition 4. A $(2 n+1)$-dimensional $(n>1) N(\kappa)$-contact metric manifold is said to be $\xi$-m-projectively flat [18] if $W^{*}(X, Y) \xi=0$ for all $X, Y \in T M$.

Theorem 5. $A(2 n+1)$-dimensional $(n>1) N(\kappa)$-contact metric manifold is $\xi$-m-projectively flat if and only if it is an $\eta$-Einstein manifold.

Proof. Let $W^{*}(X, Y) \xi=0$. Then, in view if (30), we have

$$
\begin{aligned}
R(X, Y) \xi=\frac{1}{2(n-1)}[ & S(Y, \xi) X-S(X, \xi) Y \\
& +g(Y, \xi) Q X-g(X, \xi) Q Y] .
\end{aligned}
$$

By virtue of (9), (23), and (28), the above equation reduces to

$$
\frac{1}{2}[\eta(Y) Q X-\eta(X) Q Y]-\kappa[\eta(Y) X-\eta(X) Y]=0 \text {, }
$$

which by putting $Y=\xi$, gives

$$
\mathrm{Q} X=2 \kappa[-X+(n+1) \eta(X) \xi] .
$$

Now, taking the inner product of above equation with $U$, we get

$$
S(X, U)=2 \kappa[-g(X, U)+(n+1) \eta(X) \eta(U)],
$$

which shows that $N(\kappa)$-contact metric manifold is an $\eta$ Einstein manifold. Conversely, suppose that (47) is satisfied. Then, by virtue of (46) and (31), we have $W^{*}(X, Y) \xi=0$. This completes the proof.

\section{M-Projectively Recurrent $N(\kappa)$-Contact Metric Manifolds}

Definition 6. A nonflat Riemannian manifold $M^{2 n+1}$ is said to be $\mathrm{m}$-projectively recurrent if its $\mathrm{m}$-projective curvature tensor $W^{*}$ satisfies the condition

$$
\nabla W^{*}=A \otimes W^{*},
$$

where $A$ is nonzero 1 -form. 
Theorem 7. If an $N(\kappa)$-contact metric manifold is $m$ projectively recurrent, then it is an $\eta$-Einstein manifold.

Proof. We define a function $f^{2}=g\left(W^{*}, W^{*}\right)$ on $M^{2 n+1}$, where the metric $g$ is extended to the inner product between the tensor fields. Then, we have

$$
f(Y f)=f^{2} A(Y) \text {. }
$$

This can be written as

$$
Y f=f(A(Y)), \quad(f \neq 0) .
$$

From the above equation, we have

$$
X(Y f)-Y(X f)=\{X A(Y)-Y A(X)-A([X, Y])\} f .
$$

Since the left-hand side of the above equation is identically zero and $f \neq 0$ on $M^{2 n+1}$, then

$$
d A(X, Y)=0,
$$

that is, 1 -form $A$ is closed.

Now from

$$
\left(\nabla_{Y} W^{*}\right)(Z, U) V=A(Y) W^{*}(Z, U) V,
$$

we have

$$
\left(\nabla_{X} \nabla_{Y} W^{*}\right)(Z, U) V=\{X A(Y)+A(X) A(Y)\} W^{*}(Z, U) V .
$$

In view of (52) and (54), we have

$$
\begin{aligned}
\left(R(X, Y) \cdot W^{*}\right)(Z, U) V & =[2 d A(X, Y)] W^{*}(Z, U) V \\
& =0 .
\end{aligned}
$$

Thus by virtue of Theorem 3 , the above equation shows that $M^{2 n+1}$ is an $\eta$-Einstein manifold. This completes the proof.

\section{7. $N(\kappa)$-Contact Metric Manifolds Satisfying $W^{*} \cdot S=0$}

Theorem 8. If on an $N(\kappa)$-contact metric manifold $W^{*} \cdot S=0$, then $S(Q X, Y)=2 \kappa[(n-1) S(X Y)+2 n \kappa g(X, Y)]$.

Proof. Let $W^{*}(\xi, X) \cdot S=0$. In this case, we can write

$$
S\left(W^{*}(\xi, X) Y, Z\right)+S\left(Y, W^{*}(\xi, X) Z\right)=0 .
$$

In view of (34), the above equation reduces to

$$
\begin{aligned}
-\kappa[2 n \kappa\{g(X, Y) \eta(Z)+g(X, Z) \eta(Y)\} \\
-\{\eta(Y) S(X, Z)+\eta(Z) S(X, Y)\}] \\
+\frac{1}{2}[2 n \kappa\{S(X, Y) \eta(Z)+S(X, Z) \eta(Y)\} \\
-\{\eta(Y) S(Q X, Z)+\eta(Z) S(Q X, Y)\}]=0 .
\end{aligned}
$$

Now, putting $Z=\xi$ in above equation and using (3), (9), and (23), we get

$$
S(Q X, Y)=2 \kappa[(n-1) S(X, Y)+2 n \kappa g(X, Y)] .
$$

This completes the proof.

\section{8. $N(\kappa)$-Contact Metric Manifolds Satisfying}

$$
W^{*} \cdot R=0
$$

Theorem 9. On an $N(\kappa)$-contact metric manifold, if $W^{*} \cdot R=$ 0 , then $S(Q X, Y)=2 \kappa[(n-1) S(X, Y)+2 n \kappa g(X, Y)]$.

Proof. Suppose that $W^{*}(\xi, X) \cdot R=0$, then it can be written as

$$
\begin{aligned}
& W^{*}(\xi, X) R(Y, Z) U-R\left(W^{*}(\xi, X) Y, Z\right) U \\
& -R\left(Y, W^{*}(\xi, X) Z\right) U-R(Y, Z) W^{*}(\xi, X) U=0,
\end{aligned}
$$

which on using (33), takes the form

$$
\begin{aligned}
-\frac{\kappa}{(n-1)}[g(X, R(Y, Z) U) \xi-\eta(R(Y, Z) U) X \\
-g(X, Y) R(\xi, Z) U+\eta(Y) R(X, Z) U \\
-g(X, Z) R(Y, \xi) U+\eta(Z) R(Y, X) U \\
-g(X, U) R(Y, Z) \xi+\eta(U) R(Y, Z) X] \\
-\frac{1}{2(n-1)}\left[\begin{array}{l}
{ }^{\prime} R(Y, Z, U, Q X) \xi-\eta(R(Y, Z) U) Q X \\
-
\end{array}\right. \\
-S(X, Y) R(\xi, Z) U+\eta(Y) R(Q X, Z) U \\
-S(X, U) R(Y, Z) \xi+\eta(U) R(Y, Z) Q X] \\
=0 .
\end{aligned}
$$

Taking the inner product of above equation with $\xi$, we get

$$
\begin{aligned}
-\frac{\kappa}{(n-1)}\left[{ }^{\prime}\right. & R(Y, Z, U, X)-g(X, Y) \eta(R(\xi, Z) U) \\
& +\eta(Y) \eta(R(X, Z) U)-g(X, Z) \eta(R(Y, \xi) U) \\
& +\eta(Z) \eta(R(Y, X) U)-g(X, U) \eta(R(Y, Z) \xi) \\
& +\eta(U) \eta(R(Y, Z) X)] \\
-\frac{1}{2(n-1)}[ & { }^{\prime} R(Y, Z, U, Q X)-S(X, Y) \eta(R(\xi, Z) U) \\
& +\eta(Y) \eta(R(Q X, Z) U)-S(X, Z) \eta(R(Y, \xi) U) \\
& +\eta(Z) \eta(R(Y, Q X) U)-S(X, U) \eta(R(Y, Z) \xi) \\
& +\eta(U) \eta(R(Y, Z) Q X)]=0 .
\end{aligned}
$$

Now using (22), (28), and (29) in the above equation, we get

$$
\begin{gathered}
-\frac{\kappa}{(n-1)}\left[{ }^{\prime} R(Y, Z, U, X)+\kappa\{g(X, Z) g(Y, U)\right. \\
-g(X, Y) g(Z, U)\}]
\end{gathered}
$$




$$
\begin{gathered}
-\frac{1}{2(n-1)}\left[{ }^{\prime} R(Y, Z, U, Q X)+\kappa\{S(X, Z) g(Y, U)\right. \\
-S(X, Y) g(Z, U)\}]=0 .
\end{gathered}
$$

Putting $Z=U=e_{i}$ in the above equation and summing over $i, 1 \leq i \leq 2 n+1$, we get

$$
S(Q X, Y)=2 \kappa[(n-1) S(X, Y)+2 n \kappa g(X, Y)] .
$$

This completes the proof.

\section{References}

[1] S. Tanno, "The automorphism groups of almost contact Riemannian manifolds," The Tohoku Mathematical Journal, vol. 21, pp. 21-38, 1969.

[2] K. Kenmotsu, "A class of almost contact Riemannian manifolds," The Tohoku Mathematical Journal, vol. 24, pp. 93-103, 1972.

[3] G. P. Pokhariyal and R. S. Mishra, "Curvature tensors' and their relativistics significance," Yokohama Mathematical Journal, vol. 18, pp. 105-108, 1970.

[4] R. H. Ojha, "M-projectively flat Sasakian manifolds," Indian Journal of Pure and Applied Mathematics, vol. 17, no. 4, pp. 481484, 1986.

[5] S. K. Chaubey and R. H. Ojha, "On the $m$-projective curvature tensor of a Kenmotsu manifold," Differential Geometry, vol. 12, pp. 52-60, 2010.

[6] R. N. Singh, S. K. Pandey, and G. Pandey, "On a type of Kenmotsu manifold," Bulletin of Mathematical Analysis and Applications, vol. 4, no. 1, pp. 117-132, 2012.

[7] J. P. Singh, "On m-projective recurrent Riemannian manifold," International Journal of Mathematical Analysis, vol. 6, no. 24, pp. 1173-1178, 2012.

[8] J.-B. Jun, I. B. Kim, and U. K. Kim, "On 3-dimensional almost contact metric manifolds," Kyungpook Mathematical Journal, vol. 34, no. 2, pp. 293-301, 1994.

[9] C. Baikoussis, D. E. Blair, and T. Koufogiorgos, "A decomposition of the curvature tensor of a contact manifold satisfying $R(X, Y) \xi=\kappa[\eta(Y) X-\eta(X) Y]$," Mathematics Technical Report, University of Ioanniana, 1992.

[10] B. J. Papantoniou, "Contact Riemannian manifolds satisfying $R(\xi, X) \cdot R=0$ and $\xi \in(\kappa, \mu)$-nullity distribution," Yokohama Mathematical Journal, vol. 40, no. 2, pp. 149-161, 1993.

[11] D. E. Blair, T. Koufogiorgos, and B. J. Papantoniou, "Contact metric manifolds satisfying a nullity condition," Israel Journal of Mathematics, vol. 91, no. 1-3, pp. 189-214, 1995.

[12] E. Boeckx, "A full classification of contact metric $(\kappa, \mu)$-spaces," Illinois Journal of Mathematics, vol. 44, no. 1, pp. 212-219, 2000.

[13] S. Tanno, "Ricci curvatures of contact Riemannian manifolds," The Tohoku Mathematical Journal, vol. 40, no. 3, pp. 441-448, 1988.

[14] D. E. Blair, J.-S. Kim, and M. M. Tripathi, "On the concircular curvature tensor of a contact metric manifold," Journal of the Korean Mathematical Society, vol. 42, no. 5, pp. 883-892, 2005.

[15] D. E. Blair, T. Koufogiorgos, and R. Sharma, "A classification of 3-dimensional contact metric manifolds with $Q \varphi=\varphi Q$," Kodai Mathematical Journal, vol. 13, no. 3, pp. 391-401, 1990.
[16] D. E. Blair and H. Chen, "A classification of 3-dimensional contact metric manifolds with $Q \varphi=\varphi Q$. II," Bulletin of the Institute of Mathematics, vol. 20, no. 4, pp. 379-383, 1992.

[17] U. C. De and A. Sarkar, "On a type of P-Sasakian manifolds," Mathematical Reports, vol. 11(61), no. 2, pp. 139-144, 2009.

[18] G. Zhen, J. L. Cabrerizo, L. M. Fernández, and M. Fernández, "On $\xi$-conformally flat contact metric manifolds," Indian Journal of Pure and Applied Mathematics, vol. 28, no. 6, pp. 725-734, 1997. 


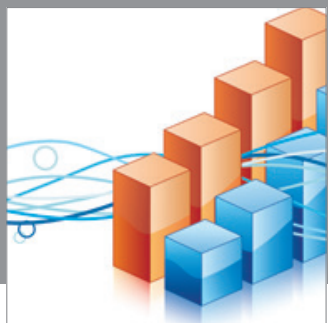

Advances in

Operations Research

mansans

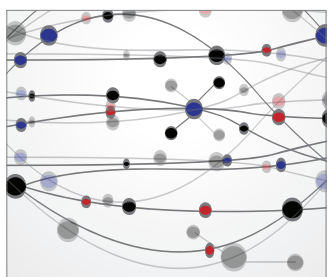

The Scientific World Journal
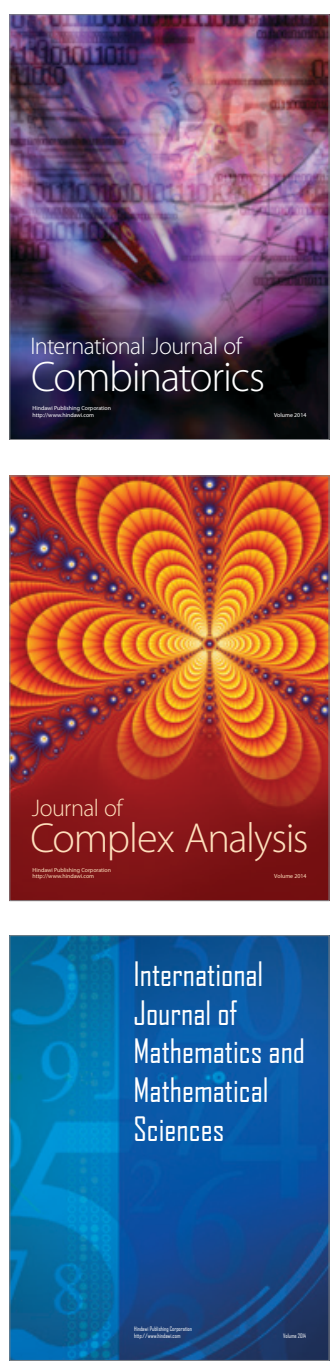
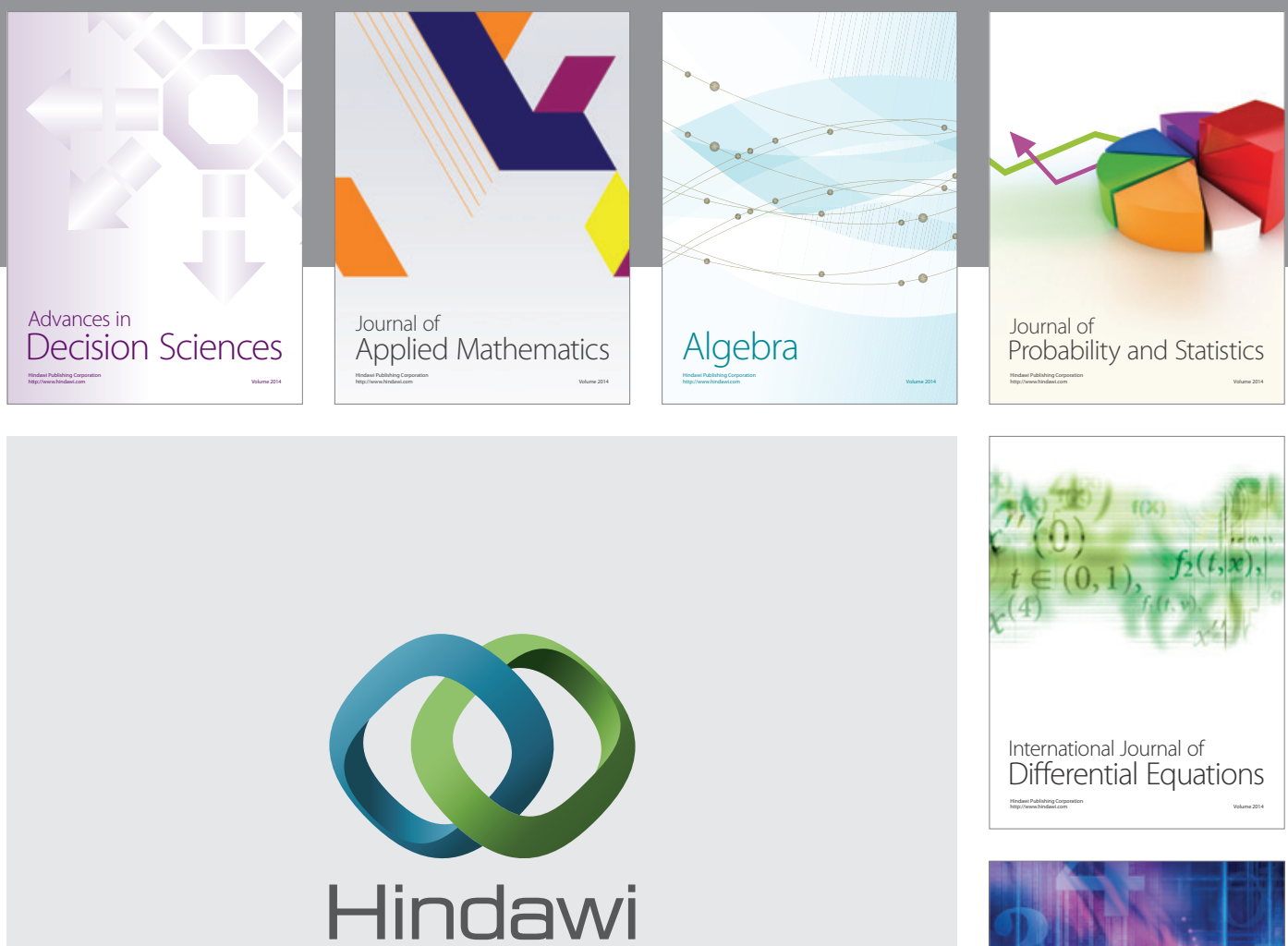

Submit your manuscripts at http://www.hindawi.com
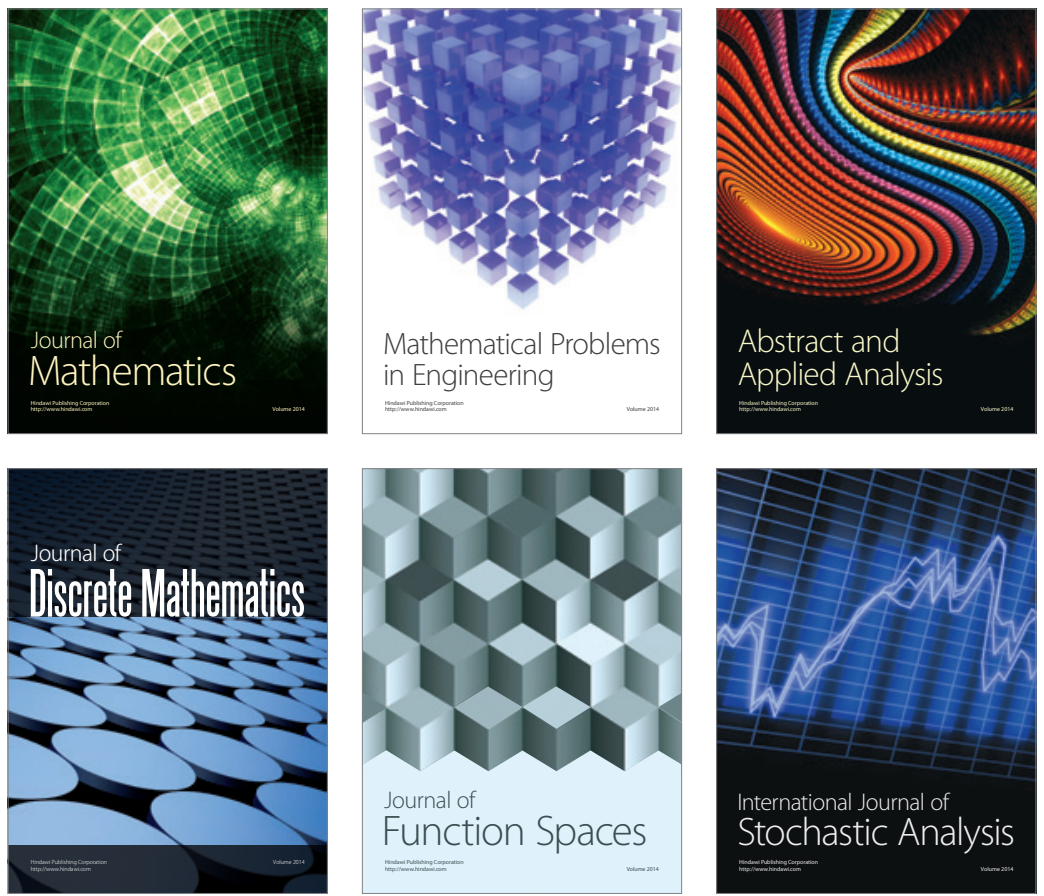

Journal of

Function Spaces

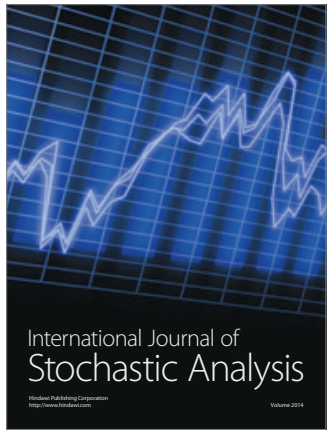

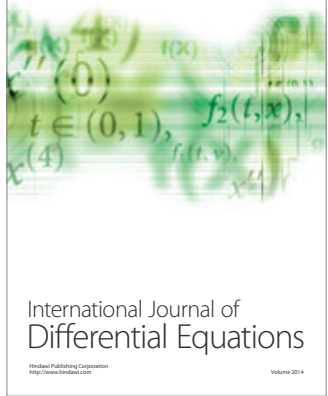
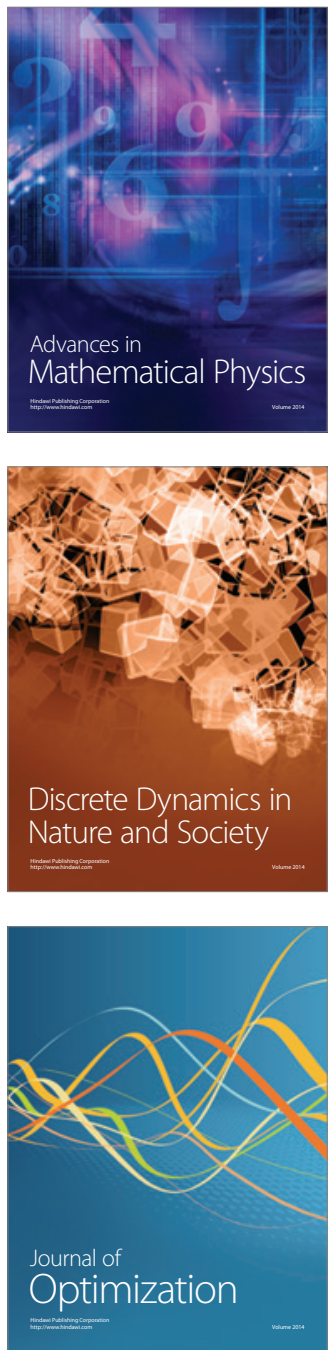